\title{
EFFECT OF MECHANICAL STIMULI ON THE SENSITIVITY OF MIMOSA PUDICA PLANT
}

\author{
Yatheesh K. Rawgol*t, Minu Mary Mathew*, \\ Bindu Thomas*, Vidya V.*, and Rinchen W.*
}

\begin{abstract}
'Touch me not' plant is a herb that shows sensation on fouch, which is taxonomically called as Mimosa pudica. The fern like leaves close up and droop when fouched; usually re-open within few minutes as o response to stimuli. Mimosa pudica reacts to mechanical stimuli with help of mechanosensitive channels. They are ideal transducers of physiologically relevant mechanical forces. The present study focus on the effect of mechanical stimuli in terms of various touch viz., human, plastic, cotton, fiber and wood on the sensitivity of Mimosa pudica plant whose leaves are at tender stage. Time taken for the leaflets to close and reopen was noted for each of the above mentioned stimuli. The t-test has shown the significant result for various mechanical stimuli. The rate of sensitivity was very high in Mimosa pudica as result of plastic touch.
\end{abstract}

Key words: Mimosa pudica, Mechano-sensitive

* School of Education, Christ University, Bangalore - 560029

† yatheesh.k@christuniversity.in (Corresponding author) 


\section{Introduction}

Mimosa pudica is well known for its rapid plant movement. In the evening the leaflets fold together and the whole leaf droops downward and later re-opens at sunrise. This type of motion has been termed nyctinastic movement. The leaves also close up under various other stimuli, such as touch, warmth and wind. The stimulus can also be transmitted to neighbouring leaves. These types of movements have been termed seismonastic movements. The movement is caused by a rapid loss of pressure in strategically situated cells that cause the leaves to droop. This characteristic is quite common within the Mimosaceae family [Singh, M.P. 2002].

Mimosa pudica reacts to mechanical stimuli by using mechano-sensitive channels. These channels are found in the cells of various types of organism - animal, plant, fungi, and bacteria. The omnipresence of these channels indicates their important physiological function in the regulation of osmolarity, cell volume, and growth. They are ideal transducers of physiologically relevant mechanical forces. Mechanosensory ion channels in plants are activated by mechanical stress and transduce the sensed information into electrical signals. In higher plants, these channels can be involved in the response to environmental stress. Plants have evolved sophisticated systems to sense environmental stimuli for adaptation as well as to sense signals from other cells for coordinated action. Plants synchronize their normal biological functions and their responses to the environment. The bio-electrochemical systems in plants not only regulate stress responses, but photosynthetic processes as well. The synchronization of internal functions based on external events is linked to the phenomenon of excitability in plant cells. The generation of electrochemical fluxes is a fundamental aspect of signal transduction. (Justin C., Talitha A. 2010).

\section{Materials and Method}

Materials required: Mimosa pudica herb, Individual (human touch), plastic, cotton, fiber, wood.

\section{Methodology of Experimentation:}

The present study was conducted at Dharmaram College, Bangalore. The study area was demarcated by the abundant growth of Mimosa herbs. The time period chosen for the experimentation were the noon hours of $2 p . m$. 
The two dependent variables taken into consideration for the study were:

a. Time taken for closure of leaflets (Initial Reading $-t_{1}$ seconds)

b. Time taken for leaflets to reopen (Final Reading $-t_{2}$ seconds)

The difference in the above two readings would yield the Recovery Time $\left(\Delta t=t_{2}-t_{1}\right)$ seconds.

Each independent variable was subjected to experimentation on the test plants and the corresponding values with respect to the dependent variables were noted. Periodic observations of the sensitivity of Mimosa pudica to the above mentioned mechanical stimuli were recorded and the time taken for recovery was noted. Human touch was used as the control.

An appropriate Mimosa pudica herb was selected. This plant $\left(\mathrm{P}_{1}\right)$ was subjected to a definite stimulus like touch. The time taken for the leaflets to close and reopen was noted and hence the recovery time was calculated. Three trials of the same test were repeated on the same plant and on two other test plants $\left(P_{1}\right.$ and $\left.P_{2}\right)$ to ensure uniformity.

The above test was followed in a similar manner using the various mechanical stimuli (plastic, cotton, fiber, wood etc.) on the same three plants as used above. In each case, the recovery time was noted and the corresponding means and standard deviations were calculated. This data was used fort-test analysis and the comparison of the validity of the stimuli was observed. Table 1 shows the statistical details of various mechanical touch.

Table 1. Statistical details of various mechanical touch

\begin{tabular}{|l|r|r|}
\hline Various Touch & \multicolumn{1}{|c|}{ S.D } & \multicolumn{1}{|c|}{ ' $t$ ' test } \\
\hline Human & 11.37 & 3.062 \\
(Control) & 13.19 & S. at 0.05 \\
Plastic & 11.37 & 15.59 \\
Human & 6.59 & S.at 0.001 \\
Cotton & 11.37 & 18.98 \\
Human & 10.79 & S.at 0.001 \\
Fibre & 11.37 & 15.296 \\
Human & 9.35 & S.at 0.001 \\
Wood &
\end{tabular}


Note: Degree of Freedom is 4. Sindicates Significance, $0.05 \& 0.001$ indicate levels of critical values

Plastic, which has pointed edge showed the significant difference at 0.05 level $(D F=4)$ when compared to the control group.

Cotton touch has shown the significant difference at 0.001 level $(D F=4)$ when compared to the control group.

Fiber which has blunt edge showed the significant difference at 0.001 level $(D F=4)$ when compared to the control group.

Wood has shown the significant difference at 0.001 level $(D F=4)$ when compared to the control group.

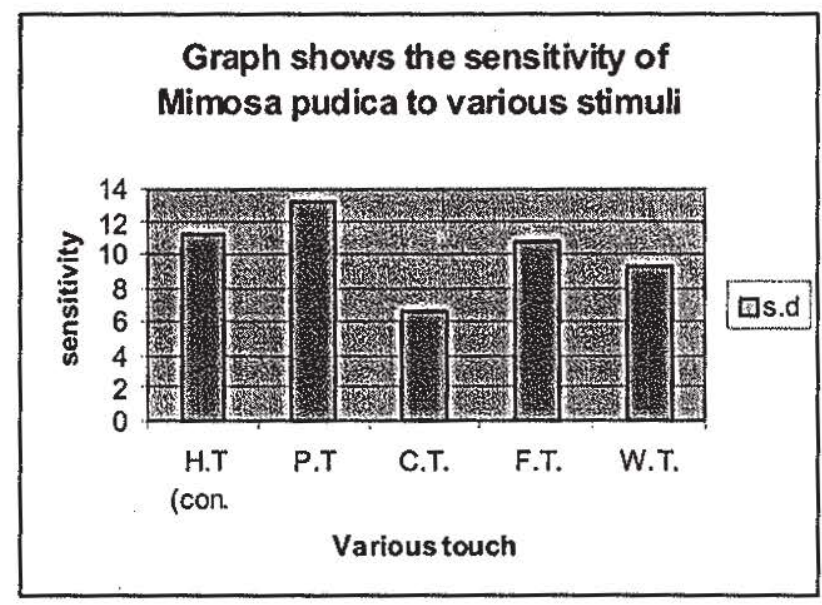

Note: $\mathrm{HT}=$ Human Touch, PT = Plastic Touch, $\mathrm{CT}=$ Cotton Touch, FT= Fiber Touch, $W T=$ Wood Touch

\section{Discussion}

The present study on the sensitivity of Mimosa pudica to mechanical stimuli has shown significant results. The plant Mimosa pudica is multicellular organism and hence necessity arises for inter communication and interaction between more or less distant organs. The evolution of a nervous system for the transmission of excitation in the living organism is a matter of much theoretical importance. Hence 
there is an influence of various stimuli on the neuro-chemical transmission of Mimosa pudica in which pulvinus acts as motile effector, the excitation being conducted along definite channels. The pulvinus of Mimosa pudica is a highly complex organs whose four quadrants act as four distinct effectors, each determining its characteristic responsive movements. The four nerve ends appear to coalesce in the pulvinus to form an almost continuous ring, in spite of which they are functionally distinct. Since in the conduction of excitation, phloem-strands in each bundle function as does a nerve. Each conducting strand is designated as plant nerve, each of these connects a particular quadrant of the pulvinous at the centre, with the corresponding sub-petiole at periphery. The continuity of the conducting channel between pulvenus and the periphery through the phloem can be made by anatomical dissections (Bose J.C. et al., 1925). The plastic touch has shown more sensitive stimuli to the Mimosa pudica compared to other mechanical stimuli viz., cotton, fiber, wood because the pointed edge probably has created more excitation in the phloem strands (nerve ends) of the vascular bundles. The cotton stimulus stands next to the plastic because of its texture. For the determination of the true impulse it is essential that the stimulus such as to produce no mechanical disturbance and its accuracy can be recorded by using resonant recorder. However, the present study did not make use of this sophisticated instrument to record the observations in depth has become one of its limitations. The impact of non-biodegradable substances such as plastics may hinder the growth of Mimosa pudica has been recognized as passing study. Other parameters which could affect the sensitivity of the plant along with the mechanical stimuli include velocity of wind and temperature of noon hours during which the trials were conducted.

\section{Conclusion}

The significance of the sensitivity of the herb Mimosa pudica to human touch, plastic, cotton, fiber and wood ascertains the fact that these can be regarded as important parameters in assessing the enigma that still revolves around this wondrous plant.

On basis of the experiment conducted by Jagdish Chandra Bose Mimosa pudica moves relatively large distances ( 1 or more inches) in short periods of time that a 20 to 1 reduction lever arm (A) must be used just to keep its movements within the range of the movement detector in a cresco-graph which was used to study the sensitivity. A long range for the sensitivity and behavior of the plant to these stimuli concludes that the experimentation holds reliable and valid on the long run. 


\section{References}

1. Bose J.C. and Das, G. Physiological and Anatomical Investigations on Mimosa pudica, 1925.

2. Justin C. Foster and Alexander G. Biologically closed electrical circuits in Mimosa pudica, Volkov Department of Chemistry and Biochemistry, Oakwood University, Huntsville, AL 35896, USA.

3. Singh, M. P. Singh, B.S. and Soma, Plant Biodiversity and Taxonomy, Daya Publishing House, 2002.

4. Talitha A. Ashby, Justin C. Foster, Ronald K. Walker, Jon A. Johnson, Deadra A. Caleb, Holly M. Carrell, Kara N. Hunter and Alexander G. Molecular Electronics of Venus flytrap and Mimosa pudica, Volkov Department of Chemistry and Biochemistry, Oakwood University, 7000.

5. http://en.wikipedia.org/wiki/Mimosa 